\title{
Sobrecualificación de los titulados universitarios y movilidad social*
}

\author{
José Saturnino Martínez García \\ Universidad de La Laguna \\ pepemart@gmail.com
}

Recepción: 26-05-2015

Aceptación: 26-05-2016

\section{Resumen}

¿La sobrecualificación de los titulados universitarios está relacionada con el origen social? Para responder a esta pregunta, se elaboran cinco indicadores de sobrecualificación, tres habituales en la literatura (objetivo, subjetivo y estadístico) y dos que permite el PIAAC (sobrecualificación estadística en competencias en lectura y numéricas). Los resultados no son concluyentes, pues sí se observa la relación en la sobrecualificación objetiva y subjetiva, pero no en el resto. Por otro lado, se recurrió a la tradición de estudios de movilidad social y se encontró que, una vez que se tiene en cuenta el nivel de estudios, el origen social influye poco en la probabilidad de lograr una ocupación de alta cualificación. Dicho de otra manera, el origen social muestra una relación intensa con el nivel de estudios alcanzado, pero la relación entre nivel de estudios y ocupación es más débil.

Palabras clave: sobrecualificación; mercado de trabajo; capital humano; movilidad social; desigualdad de oportunidades educativas; Boudon; Goldthorpe; Bourdieu; desajuste educativo

* Agradezco al Instituto Nacional de Evaluación Educativa la financiación para realizar este estudio, así como la disposición de su personal a apoyarme, en especial a Luis Sanz San Miguel, así como los comentarios de dos revisores anónimos. Este texto es una visión corregida del capítulo publicado en el tomo ir del informe PIAAC para España. También se ha recibido financiación del proyecto Ciclo económico, polarización y desigualdad, financiado por el Ministerio de Economía y Competitividad (CIEDES CSO2011-30179-C02-01), dirigido por la Dra. Olga Salido, y del International Network for Comparative Analysis of Social Inequalities, financiado por la Comisión Europea (MSCA - RISE H2020 2015 GA-691004), dirigido por el Dr. Pedro López-Roldán. Las opiniones vertidas no comprometen a las instituciones financiadoras. 


\section{Abstract. Overeducation of University Graduates and Social Mobility}

Is the overqualification of graduates related to social background? To answer this question, five indicators are developed in this paper: objective, subjective and statistical indicators common to this kind of research, and two from the PIAAC dataset, statistical overqualification in literacy and numeracy. The results are not conclusive as a relationship is observed between objective and subjective overqualification, but not the remaining indicators. From a social mobility approach, we found that once level of education is taken into account, social background has little effect on the probability of achieving a highly skilled occupation. Put another way, social origin shows an intense relationship with educational attainment, but the relationship between level of education and occupation is weaker.

Keywords: overqualification; overeducation; labor market; human capital; social mobility; inequality of educational opportunity; Boudon; Goldthorpe; Bourdieu; educational mismatch

\begin{aligned} & \multicolumn{2}{c}{ Sumario } \\ & Introducción Conclusiones \\ & Metodología Anexo \\ & Resultados Referencias bibliográficas \\ & Discusión \end{aligned}

\section{Introducción}

El debate en torno a la sobrecualificación surge en la década de 1970 en EE. UU., tras la expansión de la educación superior en la segunda mitad del siglo XX, seguida de las dificultades de los jóvenes universitarios para insertarse en el mercado de trabajo (Freeman, 1976), problema que en España llegó a atribuirse a un déficit propio de formación de la universidad (Martín Moreno y De Miguel, 1979). El objeto de investigación es resultado, por tanto, de la sensibilidad por la cuestión social de las personas que, tras haber invertido tiempo, esfuerzo y dinero en formarse, no logran un puesto de trabajo acorde a su cualificación. Este problema es mayor en España que en la mayoría de los países de la OCDE, según las estimaciones realizadas en diversos estudios (OCDE, 2013; Quintini, 2011). Se trata de un fenómeno con un recorrido histórico de décadas (Dolado et al., 2000).

La sobrecualificación se presenta tanto como un problema de frustración personal (Kucel, 2011) como un problema de tipo económico, pues aumenta la tasa de paro de equilibrio y disminuye la productividad, tanto a nivel de empresas como del país (Quintini, 2011). Las líneas que siguen resumen brevemente los principales debates teóricos en torno a la sobrecualificación a partir de la revisión de los trabajos de Sala (2011), Kucel (2011), Leuven y Oosterbeek (2011) y Quintini (2011). Luego propondremos cinco indicadores de sobrecualificación. El objeto de este estudio será averiguar si el origen social 
de las personas con estudios universitarios influye en la probabilidad de estar sobrecualificadas. Por tanto, en este trabajo, la aproximación se realiza tanto desde los estudios de sobrecualificación como desde los de movilidad social.

Desde la teoría del capital humano, el desajuste entre requerimientos formativos del puesto de trabajo y de la cualificación cabe entenderlo como provisional. La hipótesis básica de esta propuesta teórica es que los salarios son retribuidos según la productividad marginal del asalariado, por lo que, si hay desajuste, se debe al tiempo necesario para que trabajador y empresario encuentren un punto de equilibrio acorde entre productividad y salario (Becker, 1964). Ante la evidencia de que dicho desequilibrio es permanente en el tiempo, se atribuye a características no observadas del empleado que merman su productividad (Mincer, 1974). En este sentido, el salario más bajo podría deberse a que la productividad del trabajador sobrecualificado es menor que la de los que sí desempeñan un puesto de trabajo acorde a su cualificación. Esta menor productividad puede deberse a distintos factores, como un esfuerzo o una motivación inferiores del trabajador, o la heterogeneidad de los títulos educativos del mismo nivel. Algunos estudios muestran que la sobrecualificación es mayor en titulaciones universitarias de ciertas ramas que en otras, de lo cual se infiere que no es el resultado de un desajuste, sino del reconocimiento de que un título formal equivalente puede ocultar competencias laborales claramente diferentes (Barone y Ortiz, 2011). Este fenómeno, además, explicaría el incremento de la dispersión salarial entre los universitarios a medida que se amplía el catálogo de titulaciones.

Podemos interpretar como una concreción del modelo del capital humano la teoría del ajuste (Pissarides, 2000), en la que se insiste en la buena asignación entre puesto de trabajo y asalariado, debido a que el proceso de selección de personal, por parte de la empresa, y de búsqueda de trabajo adecuado, por parte del asalariado, son ambos costosos. Los empleados irían cambiando de puesto de trabajo hasta lograr un buen ajuste a su cualificación, por lo que la movilidad laboral, ya sea entre empresas o dentro de la misma empresa, será mayor en los trabajadores sobrecualificados, que todavía no han finalizado dicho ajuste.

La teoría de la señalización (Spence, 1973) considera que la formación no mejora la productividad de los trabajadores, sino que la consecución del título educativo señaliza a quienes son más productivos. El título académico es una solución a un problema de asimetría de información, pues el empresario desconoce la productividad del asalariado antes de contratarlo. Esta teoría interpreta la inversión en educación de los trabajadores de la siguiente forma: supone que existe correlación entre el rendimiento educativo y la productividad laboral, pues aquellas personas con más capacidad necesitan de menos esfuerzo para conseguir sus metas académicas, y su mayor capacidad también se hará notar en el desempeño de su ocupación. Por ello, los títulos educativos cabe interpretarlos como una señal de productividad que el trabajador "compra», con su esfuerzo, capacidad y recursos, para enviar un mensaje al empresario. Podrá existir sobrecualificación mientras los rendimientos asociados a la señal (el título educativo) sean mayores que los costes de obtenerla. En estos costes, 
debe tenerse en cuenta la capacidad del trabajador y su esfuerzo, así como las variaciones en el gasto realizado para estudiar, por lo que los cambios que tengan lugar en él (como el precio de las matrículas o los costes de oportunidad de estudiar) afectarán a la sobrecualificación, en el sentido de que será menor cuanto más caro sea adquirir cierto nivel académico.

Otra explicación se deriva del modelo de mercado laboral de Thurow (1975) y su teoría de la competición por el puesto de trabajo. Desde este punto de vista, el salario depende en mayor medida de las características del puesto de trabajo que de las características del empleado. Para este autor, existen dos colas en dicho mercado para seleccionar a la persona que ocupará una vacante en una empresa. Una es la de puestos de trabajo y la otra, la de los trabajadores, y ambas se determinan de forma diferente. Los requisitos necesarios para desempeñar adecuadamente un oficio se logran realmente en la empresa, que es donde se aprenden las habilidades necesarias. Los trabajadores se ordenan según una serie de atributos que indican que aprenderán a desempeñar sus futuras actividades laborales con más o menos dificultad. El nivel de estudios es uno de esos atributos, pero también hay otros relevantes, como la experiencia. La cola de los trabajadores está ordenada según este tipo de características, por lo que la inversión en educación no tiene tanto que ver con una mejora intrínseca de la productividad, sino con la posibilidad de mejorar la posición relativa con respecto al resto de asalariados. Como señala Sala (2011), este modelo, aunque se parece al de señalización en el mercado de trabajo (la inversión en educación no mejora la productividad del empleado), se diferencia de este en tanto que el modelo de Spence puede llegar en un momento en el cual la inversión en la señal académica se equilibra con el beneficio esperado de la educación. Sin embargo, en el modelo de Thurow, la decisión no tiene tanto que ver con la rentabilidad en sí misma, sino con la posición relativa en referencia al resto de trabajadores, por lo que es un modelo más consistente con la sobrecualificación como fenómeno permanente, a diferencia de las teorías expuestas previamente.

Otro planteamiento es la teoría de la asignación de Sattinger (1993), que tiene en cuenta tanto argumentos de la teoría del capital humano como de la competición. Según esta teoría, el salario se define tanto por la productividad de los trabajadores como por la del propio puesto de trabajo. Un puesto de trabajo determinado tendrá un techo y un suelo salarial, y en esa horquilla, la falta o el exceso de educación puede contribuir a disminuir o a aumentar la rentabilidad esperada. Esta teoría se tiene en cuenta especialmente para el análisis de la influencia del desajuste educativo sobre el rendimiento salarial.

Estas diferentes teorías dan cuenta de la existencia de la sobrecualificación, pero en esta investigación prestaremos especial atención a una cuestión más específica: en qué medida la sobrecualificación es un resultado de la desigualdad de origen socioeconómico. $\mathrm{O}$, dicho de otra forma: jla probabilidad de una persona con título universitario de ser sobrecualificada es mayor cuanto menor es el origen social de procedencia? Kucel (2010) expone esta aproximación a la sobrecualificación desde los estudios de la movilidad social, un área menos explorada que los resultados de la sobrecualificación sobre el salario, el 
bienestar psicológico o los planteamientos políticos, como señalan Marqués y Gil (2015), especialmente en los países mediterráneos. Bukodi y Goldthorpe (2011) plantean que en tanto que el fenómeno de la sobrecualificación esté vinculado al origen social y cada vez haya más personas de orígenes sociales bajos, la relación entre nivel educativo y ocupación se podría estar debilitando, con lo que invalidan la hipótesis funcionalista, según la cual la propia naturaleza del desarrollo económico y la búsqueda de la eficiencia deberían hacer que esta relación creciera con el tiempo (Treiman, 1970). Marqués y Gil (2015) repasan las teorías sobre la movilidad social para aplicarlas al estudio de la sobrecualificación y plantean que el capital económico, cultural y social de la familia de origen pueden influir en la probabilidad de sobrecualificación por distintas vías. En esta investigación, nos centraremos en el capital cultural.

El desajuste se podría estar produciendo debido a que hay ocupaciones que, además de conocimientos altamente especializados y competencias «duras» (destrezas en matemáticas y lectoescritura), exigen en su desarrollo competencias «blandas» (habilidades sociales, capacidad de liderazgo, de influencia o de autonomía, entre otras), que se forman en mayor medida en contextos de clases sociales medias y altas que de clases populares. Desde ese punto de vista, el efecto del origen social estaría mediado por la generación de características relevantes para el empresario, que percibe a unos trabajadores como más capaces de generar negocio que otros. Bourdieu (1991) ya señaló esta cuestión, incidiendo especialmente en la importancia de las afinidades de estilos de vida (de habitus de clase social) entre los trabajadores, por un lado, y los empresarios o clientes, por otro lado, en determinados sectores de actividad. Para este autor, también es importante el capital social, que facilita el acceso a información sobre el mercado de trabajo, y es más crítico con la aportación a la productividad de estos factores no cognitivos. En última instancia, no serían más que una forma de legitimar el reparto arbitrario y desigual de los recursos socioeconómicos en el seno de una sociedad.

En este sentido, hay evidencia de que la relación entre movilidad social y sobrecualificación debe hacerse teniendo en cuenta la especialidad elegida al estudiar una carrera (Torche, 2011), pues hay profesiones en las que pueden ser más relevantes destrezas cognitivas y no cognitivas que no se desarrollan en el sistema educativo. Recientemente, se ha encontrado evidencia en Italia de que la sobrecualificación está relacionada tanto con el origen social como con la titulación cursada (Capsada-Munsech, 2015).

\section{Metodología}

La fuente de datos empleada es la muestra española del Programa Internacional de Evaluación de las Competencias de la Población Adulta, conocido por sus siglas en inglés PIAAC o, de forma más popular, como el «PISA de adultos», pues mide competencias en lectura y en matemáticas y está elaborado por la OCDE (2013). El cuestionario es complejo, con gran variedad de información sobre las características laborales y educativas. La muestra es representativa de 
la población entre 16 y 65 años. La submuestra seleccionada en esta investigación es la de aquellas personas que estaban ocupadas en la semana previa a la realización de la encuesta, con edades comprendidas entre 25 y 65 años (2.886 casos). Con esta selección, se tiene en cuenta al grueso de la población que ha alcanzado su máximo nivel educativo y se evita el problema de las diferencias de competencias entre la población ocupada y no ocupada. La variable independiente principal de esta investigación es el nivel de estudios del padre, debido a que su influencia en los procesos de movilidad laboral puede ser mayor que el de la madre. Esto se debe a que, para la mayor parte de la población analizada, la tasa de actividad económica de las madres ha sido baja. Dado que la participación en el mercado de trabajo da acceso a redes sociales y a conocimientos tácitos, parece más relevante tener en cuenta la información del padre. No disponemos de su ocupación, que es una de las principales características tenidas en cuenta en los estudios de movilidad.

En cuanto a la sobrecualificación, se operacionaliza en la literatura de tres formas que reciben diferentes denominaciones. En primer lugar, la sobrecualificación normativa, de análisis del puesto de trabajo u objetiva. En segundo lugar, autoevaluación o subjetiva, y, en tercer lugar, ajuste realizado o estadistica. La definición normativa, de puesto de trabajo u objetiva consiste en definir con precisión cuáles son los requerimientos formativos del puesto de trabajo, determinando si se ajusta al nivel de formación de quien lo desempeña. Una buena aplicación de este método exige un estudio detallado de los empleos que está fuera del alcance de esta investigación, y que en otras supone disponer de un detallado catálogo de ocupaciones y de sus requerimientos formativos. Con datos más agregados, autores como García Montalvo y Peiró (2009) proponen agrupar la clasificación de ocupaciones a un dígito, de forma que puede conocerse a grandes rasgos los requerimientos de titulación de los puestos de trabajo. Este es el método seguido en la presente investigación, en la que se ha considerado que el nivel educativo de una persona está o no ajustado según la categoría ocupacional a dos dígitos de la clasificación internacional de empleos (ISCO 2008 ${ }^{1}$ ). El problema que surge al asignar un nivel de estudios a una ocupación es que produce un considerable error de medida (Glebbeek, 1993), entre otros motivos, debido a que, cuanto mayor sea el nivel de agregación de los empleos, más heterogéneo puede ser el nivel de dificultad y el tipo de competencias dentro de cada categoría.

En el método de autoevaluación (o sobrecualificación subjetiva), se pregunta a los ocupados cuáles son los requerimientos formativos de su puesto de trabajo. Esta opción plantea el inconveniente de que las personas pueden sobreestimar la dificultad de las tareas que realizan (Sloane, 2003). En cuanto a la medición estadística, toma como referencia la media de los años de escolarización de los individuos que desempeñan un determinado puesto de trabajo (u otro estadístico de tendencia central más robusto), bajo el supuesto de que esa será la formación óptima para desempeñarlo. Se considera en situación de sobrecualificación a quienes están por encima de una desviación típica, y a la

1. http://www.ilo.org/public/spanish/bureau/stat/isco/isco08/ 
inversa se trata de infracualificación. Esta forma de medición es bastante práctica, pues no supone un estudio detallado de las ocupaciones ni está sujeta a sesgos en la respuesta de los ocupados, pero no está exenta de problemas, pues la decisión de tomar como corte una desviación típica no deja de ser arbitraria. Además, con ella se corre el riesgo de conseguir un efecto de artefacto, pues pueden darse situaciones en las que muchas personas con un nivel académico alto ocupen determinados puestos de bajo requerimiento formativo, por lo que esta medición daría menos sobrecualificación de la realmente existente (o viceversa). Asimismo, la sobrecualificación dependerá de cómo sea la distribución de la escolarización en cada ocupación, pudiendo arrojar mediciones de sobrecualificación por la mera operacionalización del concepto.

Estas tres aproximaciones al estudio de la sobrecualificación comparten un mismo problema, al dar por supuesto que el nivel académico es un buen indicador de la cualificación necesaria en el puesto de trabajo. Pero, como señalan McGuinness y Wooden (2007) o Capsada-Munsech (2015), parte de los conocimientos y de las competencias adquiridas en el sistema educativo son irrelevantes desde el punto de vista empresarial. Y, por otro lado, parte de las competencias necesarias se adquieren en otros lugares — como, por ejemplo, la familia - o realizando otro tipo de actividades.

La naturaleza del estudio PIAAC nos permite aproximarnos a esta medición de la sobrecualificación de diferentes formas. Por un lado, según el método estándar, transformando el nivel educativo en años de escolarización (YRSQUAL) y realizando las operaciones pertinentes. Por otro lado, la información del PIAAC permite realizar un tratamiento novedoso, pues, además del título educativo, podemos tomar como indicadores de cualificación el nivel de competencias medido en las pruebas, que hemos elaborado para las competencias en lectura (PVLIT1) y en matemáticas (PVNUM1), siguiendo con la definición estadística de desajustes educativos (la puntuación del individuo es mayor o menor en una desviación típica según el promedio de quienes están en la ocupación).

Como señalan Flisi et al. (2016), la existencia del PIAAC permite una aproximación al análisis de la sobrecualificación, que previamente había sido poco explorada. Las investigaciones han estado dominadas por la medición de la sobrecualificación mediante el nivel de estudios, mientras que el PIAAC nos permite hacerlo mediante el nivel de competencias. Este equipo de investigación repasa diferentes operacionalizaciones. Por un lado, se puede dar una definición estadística más extrema que la que aquí proponemos $(1,5$ desviaciones típicas), por otro, se puede realizar un método mixto, como el que propone la OCDE (2013), teniendo en cuenta quién está sobrecualificado dentro de cada nivel educativo. Como señala el citado equipo, las diferentes formas de medir la sobrecualificación están correlacionadas de forma positiva, pero débil, por lo que es importante entender mejor qué estamos midiendo y comprobar diferentes tipos de mediciones, que es lo realizado en este trabajo.

En PIAAC, se pregunta a los entrevistados cuál es el nivel académico requerido para desempeñar el puesto de trabajo (D_Q12A). Esto podría generar 
cierta confusión en quienes estudiaron bajo sistemas educativos previos, pues puede que su conocimiento del sistema actual sea limitado, y ello podría generar errores. Debe tenerse en cuenta que la población investigada ha estudiado bajo tres sistemas educativos diferentes: Ley Moyano (nacidos entre 1947 y 1960), Ley general de educación (LGE, nacidos entre 1961 y 1979) y Ley Orgánica de ordenación del sistema educativo (LOGSE, nacidos entre 1985 y 1987). Los nacidos entre 1980 y 1984 estuvieron en la transición entre los dos últimos sistemas. La homogeneización de los títulos a efectos estadísticos puede ocultar características sustancialmente diferentes, entre las que cabe destacar que los ocho años de escolarización mínimos para obtener el título de graduado escolar (LGE) se han transformado en diez para asimilarlo al título de graduado en $\mathrm{ESO}^{2}$.

Los resultados de estos indicadores de desajuste educativo se presentan en la tabla 1, tomando como base la población ocupada de entre 25 y 65 años. Como es habitual en este tipo de estudios, las diferentes definiciones producen resultados divergentes de ajuste educativo. Exceptuando la sobrecualificación subjetiva (o de autoinforme), que es la que produce menos ajuste, con un $55,1 \%$, el resto oscila en un rango limitado entre el $67,6 \%$ de ajuste estadístico y el $72,4 \%$ de ajuste objetivo (el número de casos varía debido a la ausencia de información en algunas preguntas).

Tabla 1. Distribución de los distintos tipos de ajuste educativo al puesto de trabajo

\begin{tabular}{lccccc}
\hline Tipo de sobrecualificación & Infracualificado & Ajustado & Sobrecualificado & Total (\%) & Total (N) \\
\hline Sobrecualificación objetiva & $13,5 \%$ & $72,4 \%$ & $14,1 \%$ & $100,0 \%$ & 2872 \\
Subjetiva de título educativo & $24,2 \%$ & $55,1 \%$ & $20,7 \%$ & $100,0 \%$ & 2318 \\
Sobrecualificación estadística & $18,6 \%$ & $67,6 \%$ & $13,8 \%$ & $100,0 \%$ & 2878 \\
Estadística en lectura & $16,2 \%$ & $68,4 \%$ & $15,4 \%$ & $100,0 \%$ & 2885 \\
Estadística en matemáticas & $14,9 \%$ & $70,2 \%$ & $14,9 \%$ & $100,0 \%$ & 2885 \\
\hline
\end{tabular}

Fuente: población ocupada entre 25 y 65 años en los microdatos del PIAAC (OCDE, 2012).

Como se aprecia en la tabla 2 , un $73 \%$ de los sobrecualificados cumple con uno o dos requisitos, por lo que las diferentes formas de operacionalizar no convergen, un resultado habitual en este tipo de investigaciones, en que se ha

2. Cabe llamar la atención sobre dos homogeneizaciones especialmente problemáticas. Por un lado, en el paso de la Ley Moyano a la LGE, títulos de perito y otros — como de Maestro-, que se finalizaban a los 18 años o antes, pasaron a ser considerados CINE5B, es decir, equivalentes a educación superior, que se finalizaba a los 20 o 21 años. Por otro lado, en el paso de la LGE a la LOGSE, también se ha asimilado la FPII, a la que muchas personas accedían sin el bachillerato, con lo que acababan sus estudios a los 18 años, a la FP de grado superior, que se termina a los 20 años, tras haber cursado bachillerato la mayor parte del alumnado. Por tanto, homogeneizar estos títulos es dar por supuesto que dos o tres años adicionales de escolarización no producen rendimientos diferentes, ni desde el punto de vista de la productividad laboral ni desde el punto de vista de las competencias en lectura y matemáticas. 
Tabla 2. Frecuencia de coincidencia de los distintos tipos de sobrecualificación en una sola persona

\begin{tabular}{ccc}
\hline Número positivo de indicadores de sobrecualificación & Frecuencia & Porcentaje \\
\hline 1 & 426 & 44,7 \\
2 & 269 & 28,3 \\
3 & 159 & 16,7 \\
4 & 63 & 6,6 \\
5 & 33 & 3,5 \\
Total & 952 & 100 \\
\hline
\end{tabular}

Fuente: población ocupada entre 25 y 65 años en los microdatos del PIAAC (OCDE, 2012).

detectado que la correlación entre los diversos indicadores es positiva y débil (Flisi et al., 2016).

El nivel de estudios de los entrevistados es una variable decisiva en el estudio. En la tabla 3, se presenta desagregado y por nivel de estudios del padre o tutor. Apreciamos la relación entre origen social y nivel de estudios ampliamente documentada en la literatura (Martínez García, 2011; Fernández Mellizo-Soto, 2014). El porcentaje de los hijos de personas sin estudios que llegan a licenciado o máster es del $11 \%$, mientras que si el padre tiene estudios superiores, la probabilidad es de un $41,4 \%$. Por el contrario, los hijos de padres sin estudios no pasan del nivel de primarios en un $17,2 \%$, mientras que esta cifra es del 2,2\% para los hijos de padres con estudios superiores. Cabe destacar que las diferencias son menores si, en la educación superior, atendemos al nivel educativo de peritos o asimilados, diplomados e ingenieros técnicos, al que llega el $10,6 \%$ de los hijos de padres sin estudios y el $26,1 \%$ de los hijos de padres con estudios superiores.

Tabla 3. Nivel de estudios del entrevistado (desagregado) según el nivel de estudios de su padre o tutor

\begin{tabular}{lcccc}
\hline & \multicolumn{3}{c}{ Nivel de estudios del padre o tutor } \\
\cline { 2 - 5 } & $\begin{array}{l}\text { Primarios } \\
\text { o inferiores }\end{array}$ & Secundarios & Superiores & \multicolumn{1}{c}{ Total } \\
\hline Primaria o inferior (CINE 1 o menor) & $17,2 \%$ & $2,0 \%$ & $2,2 \%$ & $13,5 \%$ \\
Secundaria inferior (CINE 2, CINE 3C corta) & $25,7 \%$ & $15,0 \%$ & $3,1 \%$ & $21,8 \%$ \\
Secundaria superior (CINE 3A-B, C larga) & $22,0 \%$ & $26,4 \%$ & $14,4 \%$ & $21,8 \%$ \\
Postobligatoria, no superior (CINE 4A-B-C) & $1,7 \%$ & $0,7 \%$ & $2,8 \%$ & $1,7 \%$ \\
FP superior (CINE 5B) & $11,3 \%$ & $8,5 \%$ & $6,6 \%$ & $10,4 \%$ \\
Diplomatura, ingeniería técnica (CINE 5A1) & $10,6 \%$ & $18,3 \%$ & $26,1 \%$ & $13,3 \%$ \\
Licenciatura (CINE 5A2) & $11,0 \%$ & $27,7 \%$ & $41,4 \%$ & $16,6 \%$ \\
Doctorado (CINE 6) & $0,5 \%$ & $1,4 \%$ & $3,4 \%$ & $1,0 \%$ \\
Total & $100 \%$ & $100 \%$ & $100 \%$ & $100 \%$ \\
& $N=2184$ & $N=382$ & $N=320$ & $N=2887$ \\
\hline
\end{tabular}

Fuente: población ocupada entre 25 y 65 años en los microdatos del PIAAC (OCDE, 2012). 
Tabla 4. Tipo de ocupaciones por nivel de estudios del entrevistado

\begin{tabular}{lcrrrr}
\hline & \multicolumn{4}{c}{ Nivel de estudios (4 categorías) } \\
\cline { 2 - 6 } & $\begin{array}{l}\text { Primarios } \\
\text { o inferiores }\end{array}$ & Secundarios & FPGS / FPII & Superiores & \multicolumn{1}{c}{ Total } \\
\hline Cualificadas & $10,6 \%$ & $26,6 \%$ & $32,1 \%$ & $75,6 \%$ & $36,5 \%$ \\
Cuello blanco semicualificadas & $32,1 \%$ & $44,8 \%$ & $37,9 \%$ & $20,0 \%$ & $31,9 \%$ \\
Cuello azul semicualificadas & $34,3 \%$ & $17,5 \%$ & $24,4 \%$ & $2,0 \%$ & $19,5 \%$ \\
Ocupaciones elementales & $23,0 \%$ & $11,1 \%$ & $5,6 \%$ & $2,4 \%$ & $12,1 \%$ \\
Total & $100 \%$ & $100 \%$ & $100 \%$ & $100 \%$ & $100 \%$ \\
& $N=1044$ & $N=679$ & $N=301$ & $N=897$ & $N=2921$ \\
\hline
\end{tabular}

Fuente: población ocupada entre 25 y 65 años en los microdatos del PIAAC (OCDE, 2012).

Debido a que este nivel de detalle lleva a submuestras muy pequeñas, en la investigación posterior se ha procedido a agrupar el nivel de estudios en cuatro categorías: primarios o inferiores (Clasificación Internacional Normalizada de Educación (CINE) 1 o menor), secundarios (CINE 2, 3 o 4), formación profesional de grado superior o FP II (CINE 5B) y universitarios (CINE 5A y 6, (tabla 4)). El precio a pagar para lograr mayor tamaño muestral es el incremento de la heterogeneidad en cada nivel educativo, especialmente en la educación secundaria (CINE 2, 3 o 4) y en la académica superior (5A y 6), lo cual debe tenerse en cuenta en la interpretación de los datos. Para minimizar este problema, en los estudios universitarios, los cálculos estadísticos de sobrecualificación se hacen separando los niveles $5 \mathrm{~A} 1$ y $5 \mathrm{~A} 2$, aunque los resultados se presenten con los datos agrupados.

En la tabla 4, mostramos el tipo de ocupación desempeñada según el nivel de estudios del entrevistado, en lo que vendría a ser otra medida de ajuste entre cualificación y ocupación. Apreciamos que un 75,6\% de las personas con estudios superiores universitarios desempeñan ocupaciones cualificadas, mientras que este porcentaje el del 10,6\% en los de estudios primarios o inferiores.

El resto de variables consideradas en este estudio son el sexo, la edad, la nacionalidad y la experiencia en el puesto de trabajo, todas ellas características individuales asociadas a la sobrecualificación, para comparar su efecto con el origen social, así como el tipo de competencias asociadas a las tareas realizadas en su profesión. Los efectos del sexo tienden a apuntar mayor sobrecualificación entre las mujeres, especialmente si están casadas. Este hecho se explica debido a que las carreras laborales masculinas tienden a ser más estables y mejor remuneradas que las femeninas, por lo que ellas orientan más sus decisiones a conciliar vida laboral y familiar, mientras que ellos toman decisiones guiadas en mayor medida por cuestiones laborales. Por estos motivos, cabría esperar que, cuanto mayor sea la facilidad para conciliar, menores deberían ser las diferencias entre hombres y mujeres. En cuanto a la edad, en la medida en que está asociada a la trayectoria laboral, se espera que, cuanto mayor sea la persona, menor sobrecualificación tenga. Pero esta relación puede no ser lineal, debido a las complejas relaciones con el desarrollo a lo largo del ciclo vital de 
las capacidades cognitivas y de los efectos de periodo (Desjardins y Warnke, 2011). Por un lado, a mayor edad, menor es la inteligencia fluida, pero más amplia la cristalizada y, en la medida en que la inteligencia cristalizada vaya quedando obsoleta para resolver problemas, las personas de mayor edad, a pesar de mantener sus credenciales educativas, pueden perder cierto potencial laboral debido a la combinación entre obsolescencia de sus conocimientos y dificultad para adquirir otros nuevos. Estaríamos, por tanto, en un caso que podría quedar registrado como sobrecualificación desde el punto de vista del título educativo, pero no tanto desde el punto de vista de las competencias. En cuanto a la nacionalidad, puede estar relacionada con la sobrecualificación por tres motivos. Por un lado, la realización de ciertas ocupaciones puede tener particularidades nacionales, como, por ejemplo, el ejercicio de la abogacía. Por otro, aunque tales particularidades sean mínimas, puede haber dificultades de reconocimiento de las credenciales académicas, como sucede en el caso de la medicina. Por último, es posible que también exista discriminación. En cuanto a la experiencia en el puesto de trabajo, cabe esperar que, si el ajuste se ha producido, tanto empresario como trabajador tendrán menos incentivo para la movilidad laboral, ya sea por promoción interna o por rotación entre empresas.

En lo referente a las competencias desarrolladas en el puesto de trabajo, se han elaborado dos variables compuestas a partir de la información proporcionada por los entrevistados a los siguientes índices elaborados con las respuestas a diversas preguntas: empleo de tecnologías de la información en el trabajo (ICTWORK), si en el trabajo se influye sobre otras personas (INFLUENCE), si se necesitan competencias numéricas (NUMWORK), leer (READWORK), escribir (WRITWORK), planificar (PLANNING) o si es necesario aprender tareas nuevas (LEARNATWORK). Con estos índices, se procedió a extraer dos factores mediante componentes principales y rotarlos por el procedimiento Varimax (véase anexo). De los dos factores, el primero puede ser considerado de "competencias duras", vinculado especialmente a trabajar con TIC, realizando cálculos, leyendo y escribiendo informes. El segundo factor lo podemos considerar de "competencias blandas», por estar asociado a la planificación, a las relaciones sociales y al aprendizaje. El método de extracción de los factores conduce a que estén incorrelados, sean adimensionales y se distribuyan como una normal canónica (es decir, media cero y varianza, la unidad).

\section{Resultados}

En la tabla 5, se presenta la relación entre origen social y tasa de sobrecualificación. A medida que el nivel educativo del padre es mayor, menor es la sobrecualificación, tanto la subjetiva como la objetiva. Sin embargo, en las tres definiciones estadísticas, no se observa la relación esperada. En el caso de los años de escolarización, las diferencias son pequeñas, mientras que, en el caso de las diferencias en competencias, son claramente contrarias a las esperadas, con mayor tasa de sobrecualificación a medida que es mayor el nivel de estudios del padre o tutor. Como veremos más adelante (tabla 15), esto puede deberse 
Tabla 5. Nivel de estudios del padre o tutor y tasa de sobrecualificación de los titulados universitarios y del conjunto de la población (25-65 años)

\begin{tabular}{llcccc}
\hline & & \multicolumn{3}{c}{ Nivel de estudios del padre o tutor } \\
\cline { 3 - 6 } & & $\begin{array}{c}\text { Primarios } \\
\text { o inferiores }\end{array}$ & Secundarios & Superiores & Total \\
\hline Universitarios & Objetiva & 28,3 & 25,8 & 19,9 & 25,7 \\
& Subjetiva & 41,2 & 37,9 & 31,6 & 38,2 \\
& Estadística (años de escolarización) & 28,7 & 32,8 & 27,0 & 29,1 \\
& Estadística en lectura & 23,2 & 21,8 & 30,4 & 24,8 \\
& Estadística en matemáticas & 20,3 & 24,1 & 27,8 & 23,0 \\
\hline Total & Objetiva & 12,8 & 17,3 & 17,5 & 14,0 \\
& Subjetiva & 18,9 & 26,7 & 30,5 & 21,3 \\
& Estadística (años de escolarización) & 11,5 & 19,0 & 22,9 & 13,8 \\
& Estadística en lectura & 13,0 & 21,1 & 28,2 & 15,7 \\
& Estadística en matemáticas & 13,4 & 22,0 & 24,9 & 15,8 \\
\hline
\end{tabular}

Fuente: población ocupada entre 25 y 65 años en los microdatos del PIAAC (OCDE, 2012).

a que, a igualdad de titulación, el nivel de competencias de las personas con un padre de alto nivel educativo es mayor, y la diferencia es más elevada en los niveles educativos más bajos de los entrevistados. En tanto que muchos puestos de trabajo se deciden por la titulación formal, las personas de origen alto con baja titulación no harían valer en este punto su mejor nivel de competencias. Este hallazgo es coherente con las teorías de la señalización y del credencialismo, pero no con la del capital humano, pues muestra que el mercado laboral tiende a reconocer los títulos educativos en vez de las competencias. Si, en vez de tomar solo los universitarios, tomamos el conjunto de la población, la relación es contraria a la esperada, pero esto se debe a un efecto de composición, pues la sobrecualificación es menor cuanto más bajo es el nivel académico del entrevistado.

En cuanto a las diferencias entre hombres y mujeres (tabla 6), tampoco hay un patrón común para las diferentes definiciones de sobrecualificación. Entre los titulados universitarios, la sobrecualificación de hombres y mujeres es muy similar si la definimos como objetiva o estadística en años de escolarización, pero es mayor para los varones cuando se define como subjetiva o por competencias, tanto en lectura como en matemáticas. También se intentó comprobar la sobrecualificación de la población que obtuvo su máximo nivel educativo en el extranjero. En general, no se observaron grandes diferencias con el resto de la muestra, pero, dado el escaso número de observaciones (menos de treinta), se ha preferido omitir los resultados.

La experiencia laboral es la única característica que muestra un patrón coherente en todos los indicadores de sobrecualificación (tabla 8) en el sentido esperado: a más antigüedad en el puesto, menos sobrecualificación, aunque en algunos casos la mayor diferencia está entre 0-3 años (duración máxima de los contratos temporales) y el resto de experiencia. Cabrales et al. (2013) 
Tabla 6. Tasa de sobrecualificación según sexo, para los titulados universitarios y para el conjunto de la población

\begin{tabular}{llccc}
\hline & & \multicolumn{3}{c}{ Sexo } \\
\cline { 3 - 5 } & & Hombre & Mujer & Total \\
\hline Universitarios & Objetiva & 26,1 & 25,3 & 25,7 \\
& Subjetiva & 42,3 & 35,3 & 38,4 \\
& Estadística (años de escolarización) & 28,6 & 29,4 & 29,0 \\
& Estadística en lectura & 29,5 & 19,9 & 24,4 \\
& Estadística en matemáticas & 30,2 & 15,9 & 22,7 \\
\hline Total & Objetiva & 13,6 & 14,1 & 13,9 \\
& Subjetiva & 18,8 & 23,9 & 21,2 \\
& Estadística (años de escolarización) & 13,0 & 14,4 & 13,6 \\
& Estadística en lectura & 17,2 & 13,5 & 15,6 \\
& Estadística en matemáticas & 19,0 & 11,5 & 15,6 \\
\hline
\end{tabular}

Fuente: población ocupada entre 25 y 65 años en los microdatos del PIAAC (OCDE, 2012).

encuentran una clara relación negativa entre el tipo de contratación y el nivel de competencias. Este resultado es coherente con la teoría del ajuste, en el sentido de que la sobrecualificación debe disminuir con el paso del tiempo, pues, como su propio nombre indica, es un proceso de ajuste laboral.

En cuanto a la edad (tabla 9), la sobrecualificación de los titulados universitarios sigue una evolución en $U$ invertida en todos los tipos de sobrecualificación excepto en la objetiva. Este patrón es similar al observado en la evolución a lo largo de la edad de las competencias cognitivas, pues las denominadas inteligencia fluida (capacidad de innovar) y cristalizada (capacidad de hacer uso de la experiencia) evolucionan de forma distinta a lo largo del ciclo vital, y la combinación álgida de ambas se produce en las edades medias.

Tabla 7. Tasa de sobrecualificación según experiencia en el empleo, para la población universitaria y para el total

\begin{tabular}{llcccc}
\hline & & \multicolumn{3}{c}{ Experiencia en el empleo actual } \\
\cline { 2 - 5 } $\begin{array}{l}\text { Nivel de estudios } \\
\text { del entrevistado }\end{array}$ & Tipo de sobrecualificación & \multicolumn{4}{c}{9 y más } \\
\hline Universitarios & Objetiva & 33,3 & 25,2 & 22,5 & 26,0 \\
& Subjetiva & 48,2 & 37,6 & 33,7 & 38,5 \\
& Estadística (años de escolarización) & 39,7 & 27,2 & 26,2 & 30,0 \\
& Estadística en lectura & 29,1 & 25,9 & 22,1 & 24,8 \\
& Estadística en matemáticas & 26,3 & 22,4 & 21,4 & 22,9 \\
\hline Total & Objetiva & 16,3 & 14,1 & 13,4 & 14,4 \\
& Subjetiva & 25,3 & 22,0 & 18,3 & 21,2 \\
& Estadística (años de escolarización) & 17,8 & 12,0 & 12,6 & 13,9 \\
& Estadística en lectura & 18,4 & 15,3 & 15,0 & 16,0 \\
& Estadística en matemáticas & 16,9 & 16,8 & 15,2 & 16,1 \\
\hline
\end{tabular}

Fuente: población ocupada entre 25 y 65 años en los microdatos del PIAAC (OCDE, 2012). 
Tabla 8. Tasa de sobrecualificación por grupo de edad, para los titulados universitarios y para el conjunto de la población

\begin{tabular}{llccccc}
\hline & & \multicolumn{4}{c}{ Grupos de edad } & \\
\cline { 3 - 6 } Nivel de estudios & & & & & \\
del entrevistado & Tipo de sobrecualificación & $25-34$ & $35-44$ & $45-54$ & $55-65$ & Total \\
\hline Universitarios & Objetiva & 27,5 & 29,3 & 19,2 & 25,1 & 25,7 \\
& Subjetiva & 40,2 & 42,2 & 35,8 & 27,8 & 38,4 \\
& Estadística & 30,1 & 31,7 & 26,0 & 24,7 & 29,0 \\
& Estadística en lectura & 22,5 & 28,6 & 24,8 & 14,7 & 24,4 \\
& Estadística en matemáticas & 21,1 & 26,2 & 23,7 & 12,1 & 22,7 \\
\hline Total & Estadística objetiva & 16,8 & 16,9 & 10,4 & 8,4 & 13,9 \\
& Subjetiva & 24,5 & 23,4 & 19,8 & 10,6 & 21,2 \\
& Estadística & 16,3 & 16,0 & 11,3 & 8,0 & 13,6 \\
& Estadística en lectura & 16,2 & 19,4 & 14,7 & 7,2 & 15,6 \\
& Estadística en matemáticas & 17,7 & 19,3 & 13,8 & 7,1 & 15,6 \\
\hline
\end{tabular}

Fuente: población ocupada entre 25 y 65 años en los microdatos del PIAAC (OCDE, 2012).

Aparte de estas definiciones estándar, hemos procedido a comprobar qué tipo de competencias se demandaban en cada ocupación, a partir de las puntuaciones de los factores que se muestran en el anexo. En la tabla 10, observamos el promedio del primer factor (que se distribuye como una normal canónica), que condensa la información del conjunto de requerimientos del puesto de trabajo, especialmente los relacionados con las competencias duras. Apreciamos que las personas con un padre que posee un nivel de estudios universitarios dicen estar en puestos de mayor requerimiento de competencias, a igualdad de nivel de estudios de los entrevistados. Las diferencias con respecto al promedio están entre 0,05 y 0,2 desviaciones típicas, por lo que no son demasiado altas.

En la tabla 11, apreciamos los resultados para el factor que recoge competencias «blandas». Vemos que, para los individuos con título educativo bajo (estudios secundarios o inferiores), el nivel de exigencias no cognitivas del puesto está relacionado positivamente con el nivel educativo del padre. Sin embargo,

Tabla 9. Media de la puntuación del factor de exigencias de competencias «duras»y genéricas del puesto de trabajo, según el nivel de estudios del padre y del entrevistado

\begin{tabular}{lcccr}
\hline & \multicolumn{3}{c}{ Nivel de estudios del padre o tutor } \\
\cline { 2 - 5 } Nivel de estudios del entrevistado & $\begin{array}{c}\text { Primarios } \\
\text { o inferiores }\end{array}$ & Secundarios & Superiores & \multicolumn{1}{c}{ Total } \\
\hline Primarios o sin estudios & $-0,28$ & $-0,15$ & 0,19 & $-0,27$ \\
Secundarios & $-0,02$ & 0,02 & 0,17 & 0 \\
FPGS/FP II & $-0,1$ & $-0,12$ & $-0,22$ & $-0,11$ \\
Universitarios & 0,42 & 0,41 & 0,34 & 0,4 \\
Total & $-0,04$ & 0,16 & 0,27 & 0,02 \\
\hline
\end{tabular}

Fuente: población ocupada entre 25 y 65 años en los microdatos del PIAAC (OCDE, 2012). 
Tabla 10. Media de la puntuación del factor de exigencias de competencias «blandas»y genéricas del puesto de trabajo, según el nivel de estudios del padre y del entrevistado

\begin{tabular}{lcccr}
\hline & \multicolumn{3}{c}{ Nivel de estudios del padre o tutor } \\
\cline { 2 - 5 } Nivel de estudios del entrevistado & $\begin{array}{c}\text { Primarios } \\
\text { o inferiores }\end{array}$ & Secundarios & Superiores & \multicolumn{1}{c}{ Total } \\
\hline Primarios o sin estudios & $-0,29$ & $-0,15$ & 0,13 & $-0,28$ \\
Secundarios & $-0,03$ & 0,02 & 0,08 & 0 \\
FPGS/FP II & $-0,07$ & 0,03 & $-0,19$ & $-0,07$ \\
Universitarios & 0,43 & 0,41 & 0,37 & 0,41 \\
Total & $-0,06$ & 0,19 & 0,27 & 0,01 \\
\hline
\end{tabular}

Fuente: población ocupada entre 25 y 65 años en los microdatos del PIAAC (OCDE, 2012).

cuando los estudios son superiores, a mayor nivel educativo del padre, menores son dichas competencias. Esto quizá podría deberse a un sesgo de selección, en el sentido de que las personas de origen social bajo que logran un título universitario podrían estar más seleccionadas en factores no cognitivos que las personas de origen social alto. Con este mejor nivel de competencias blandas, habrían podido sortear las mayores dificultades encontradas en el desarrollo de su carrera educativa y profesional. Esto parece contradecir el argumento extendido en la literatura citada en la introducción, según el cual las personas de origen social alto destacarían más en competencias no cognitivas, por lo que serán necesarias posteriores investigaciones para indagar esta cuestión.

Otra forma de buscar posibles diferencias en sobrecualificación es averiguar cuál es el número medio de años de escolarización de las ocupaciones en las cuales trabajan los entrevistados (otra forma de aproximarse a la sobrecualificación estadística). Como vemos en la tabla 12, no se aprecian diferencias relevantes, es decir, las personas que han alcanzado cierto nivel educativo están en ocupaciones en las que el promedio de escolarización es similar. Cabe destacar que la diferencia en los años medios de escolarización entre los cuatro niveles educativos es de cuatro cursos (10,6 a 14,4), lo que, en términos absolutos, no es una cifra considerable.

Tabla 11. Media de años de escolarización de la ocupación por nivel educativo del progenitor y del entrevistado

\begin{tabular}{lcccc}
\hline & \multicolumn{3}{c}{ Nivel de estudios del padre o tutor } \\
\cline { 2 - 5 } Nivel de estudios del entrevistado & 10,6 & 11,1 & & Primarios \\
o inferiores & Secundarios & Superiores & Total \\
\hline Primarios o sin estudios & 11,8 & 12,0 & 10,8 & 10,6 \\
Secundarios & 12,1 & 12,4 & 12,3 & 11,8 \\
FPGS/FP II & 14,3 & 14,3 & 14,6 & 14,4 \\
Universitarios & 11,9 & 13,0 & 13,8 & 12,2 \\
\hline Total &
\end{tabular}

Fuente: población ocupada entre 25 y 65 años en los microdatos del PIAAC (OCDE, 2012). 
Tabla 12. Nivel de estudios alcanzado por el entrevistado (en \%), según el nivel de estudios del padre o tutor

\begin{tabular}{lccccccc}
\hline & \multicolumn{9}{c}{ Nivel de estudios del entrevistado } & & \\
\cline { 2 - 5 } $\begin{array}{l}\text { Nivel de estudios } \\
\text { del padre o tutor }\end{array}$ & $\begin{array}{c}\text { Primarios } \\
\text { o inferiores }\end{array}$ & Secundarios & FPGS/FP II & Universitarios & & \multicolumn{2}{c}{ Total } \\
\hline Primarios o inferiores & $42,9 \%$ & $23,7 \%$ & $11,3 \%$ & $22,0 \%$ & & $100 \%$ & 2184 \\
Secundarios & $17,0 \%$ & $27,1 \%$ & $8,5 \%$ & $47,3 \%$ & & $100 \%$ & 382 \\
Superiores & $5,3 \%$ & $17,2 \%$ & $6,6 \%$ & $70,9 \%$ & & $100 \%$ & 320 \\
Total & $35,3 \%$ & $23,5 \%$ & $10,4 \%$ & $30,8 \%$ & & $100 \%$ & 2887 \\
\hline
\end{tabular}

Fuente: población ocupada entre 25 y 65 años en los microdatos del PIAAC (OCDE, 2012).

Dada la falta de resultados claros mediante estos procedimientos, se ha procedido a estudiar la relación entre el origen social, el nivel de estudios y la ocupación concentrada en grandes grupos, más habitual en los estudios de movilidad social. Comenzando por la relación entre origen social y logro educativo (tabla 13, en la que se presenta reunida la información de la tabla 3), vemos la fuerte relación entre ambos, tal y como ha quedado reflejado repetidamente en la investigación social (Fernández Mellizo-Soto, 2014). La probabilidad de lograr un nivel de estudios universitarios es del $70,9 \%$ para los hijos de universitarios, mientras que es de un $22 \%$ para los hijos de personas con nivel académico bajo. En cuanto a la probabilidad de no pasar del nivel de estudios primarios, es del 5,3 y del 42,9\%, respectivamente.

En la tabla 14, se muestra la probabilidad de alcanzar un cierto nivel ocupacional (en porcentaje) según el nivel educativo del padre y del entrevistado, en lo que es una típica tabla de análisis de movilidad social. En el total, apreciamos una notable influencia entre el origen social y el destino ocupacional: la probabilidad de lograr una ocupación cualificada varía considerablemente dependiendo del nivel educativo del progenitor, hasta el punto de que la probabilidad de desempeñarla es de más del doble para quienes provienen de una familia con un padre con estudios universitarios, frente a quienes provienen de una familia con un nivel de estudios bajo (un 68\% y un 32\%, respectivamente), mientras que la probabilidad de desempeñar una ocupación elemental es casi tres veces mayor para quienes provienen de familias de nivel educativo bajo que de nivel alto (un 14\% y un 5\%, respectivamente).

Esta desigualdad de oportunidades opera fundamentalmente mediante el sistema educativo, pues apreciamos que las diferencias dentro de cada nivel académico son relativamente pequeñas y aparecen en el sentido predicho por las teorías de la movilidad social. En los niveles educativos medios de los entrevistados, prácticamente no hay desigualdad de oportunidades por origen social, pero donde más se hace notar esta influencia es en los niveles educativos más alto y más bajo. Por un lado, si los entrevistados tienen el nivel de estudios más bajo, la probabilidad de lograr una ocupación cualificada es casi el doble para quienes tienen padres universitarios frente a quienes tienen 
Tabla 13. Tipo de ocupación alcanzada por el entrevistado (en \%), según el nivel educativo del padre o tutor

\begin{tabular}{|c|c|c|c|c|c|c|c|}
\hline \multirow[b]{2}{*}{$\begin{array}{l}\text { Nivel de estudios } \\
\text { del entrevistado }\end{array}$} & \multirow[b]{2}{*}{$\begin{array}{l}\text { Nivel de estudios } \\
\text { del padre } 0 \text { tutor }\end{array}$} & \multicolumn{4}{|c|}{ Tipo de ocupación del entrevistado } & \multirow{2}{*}{\multicolumn{2}{|c|}{ Total (\%) Total (N) }} \\
\hline & & Cualificadas & $\begin{array}{l}\text { Semicualificadas } \\
\text { de cuello blanco }\end{array}$ & $\begin{array}{l}\text { Semicualificadas } \\
\text { de cuello azul }\end{array}$ & $\begin{array}{l}\text { Ocupaciones } \\
\text { elementales }\end{array}$ & & \\
\hline \multirow{4}{*}{$\begin{array}{l}\text { Primarios } \\
0 \text { inferiores }\end{array}$} & Primarios o inferiores & $10,5 \%$ & $31,3 \%$ & $34,7 \%$ & $24,0 \%$ & $100 \%$ & 930 \\
\hline & Secundarios & $14,6 \%$ & $46,0 \%$ & $30,8 \%$ & $9,0 \%$ & $100 \%$ & 65 \\
\hline & Superiores & $19,9 \%$ & $32,0 \%$ & $13,6 \%$ & $35,0 \%$ & $100 \%$ & 17 \\
\hline & Total & $10,9 \%$ & $32,3 \%$ & $34,1 \%$ & $22,7 \%$ & $100 \%$ & 1013 \\
\hline \multirow[t]{4}{*}{ Secundarios } & Primarios 0 inferiores & $26,1 \%$ & $44,1 \%$ & $19,4 \%$ & $10,0 \%$ & $100 \%$ & 516 \\
\hline & Secundarios & $29,8 \%$ & $44,9 \%$ & $12,5 \%$ & $13,0 \%$ & $100 \%$ & 102 \\
\hline & Superiores & $29,1 \%$ & $46,4 \%$ & $10,3 \%$ & $14,0 \%$ & $100 \%$ & 55 \\
\hline & Total & $26,9 \%$ & $44,4 \%$ & $17,6 \%$ & $11,1 \%$ & $100 \%$ & 673 \\
\hline \multirow[t]{4}{*}{ FPGS/FP II } & Primarios o inferiores & $32,1 \%$ & $36,8 \%$ & $24,9 \%$ & $6,0 \%$ & $100 \%$ & 246 \\
\hline & Secundarios & $33,4 \%$ & $46,7 \%$ & $18,2 \%$ & $2,0 \%$ & $100 \%$ & 33 \\
\hline & Superiores & $30,6 \%$ & $39,5 \%$ & $24,5 \%$ & $5,0 \%$ & $100 \%$ & 21 \\
\hline & Total & $32,1 \%$ & $38,0 \%$ & $24,2 \%$ & $5,6 \%$ & $100 \%$ & 300 \\
\hline \multirow[t]{4}{*}{ Universitarios } & Primarios o inferiores & $71,7 \%$ & $23,3 \%$ & $1,5 \%$ & $4,0 \%$ & $100 \%$ & 480 \\
\hline & Secundarios & $74,9 \%$ & $20,9 \%$ & $2,1 \%$ & $2,0 \%$ & $100 \%$ & 179 \\
\hline & Superiores & $84,6 \%$ & $12,5 \%$ & $2,5 \%$ & $0,0 \%$ & $100 \%$ & 225 \\
\hline & Total & $75,6 \%$ & $20,1 \%$ & $1,9 \%$ & $2,4 \%$ & $100 \%$ & 885 \\
\hline \multirow[t]{4}{*}{ Total } & Primarios o inferiores & $30,2 \%$ & $33,2 \%$ & $22,6 \%$ & $14,0 \%$ & $100 \%$ & 2173 \\
\hline & Secundarios & $48,9 \%$ & $33,9 \%$ & $11,2 \%$ & $6,0 \%$ & $100 \%$ & 379 \\
\hline & Superiores & $68,0 \%$ & $21,2 \%$ & $5,9 \%$ & $5,0 \%$ & $100 \%$ & 318 \\
\hline & Total & $36,8 \%$ & $32,0 \%$ & $19,3 \%$ & $12,0 \%$ & $100 \%$ & 2870 \\
\hline
\end{tabular}

Fuente: población ocupada entre 25 y 65 años en los microdatos del PIAAC (OCDE, 2012).

padres sin estudios (un 19,9\% versus un 10,5\%), por lo que, desde este punto de vista, la infracualificación es mayor en las personas de origen social alto. Por otro lado, entre los individuos con estudios universitarios superiores, quienes provienen de familias con un nivel educativo más elevado tienen mayor probabilidad de estar «ajustados» en ocupaciones cualificadas que quienes se han criado en familias de nivel educativo más bajo (un $84,6 \%$ y un $71,5 \%$, respectivamente).

Con estos resultados, la relación entre origen social, logro educativo y trayectoria profesional muestra que el origen social opera especialmente a través de las diferencias en la probabilidad de acceso a cierto nivel académico, pero, una vez que se obtiene el título, la influencia del origen social es pequeña. Dicho de otra forma, la desigualdad de oportunidades por origen social es fuerte en educación y mediada por ésta en la vida laboral.

La probabilidad diferente de alcanzar una ocupación de cierto nivel, ¿hasta qué punto depende de un nivel de competencias no reflejado en el título educativo? Pues puede que sí dependa, aunque hay que ser cauto en el análisis de los datos, pues las submuestras son de tamaño muy pequeño (como se aprecia en los totales de la tabla anterior). Para evitar este problema, nos centramos 
Tabla 14. Puntuación media de competencias en lectura según el nivel de estudios del entrevistado, su ocupación y el nivel de estudios de su padre o tutor

\begin{tabular}{|c|c|c|c|c|c|c|}
\hline \multirow[b]{2}{*}{$\begin{array}{l}\text { Nivel de estudios } \\
\text { del entrevistado }\end{array}$} & \multirow[b]{2}{*}{$\begin{array}{l}\text { Nivel de estudios } \\
\text { del padre o tutor }\end{array}$} & \multicolumn{4}{|c|}{ Tipo de ocupación del entrevistado } & \multirow[b]{2}{*}{ Total } \\
\hline & & Cualificadas & $\begin{array}{l}\text { Semicualificadas } \\
\text { de cuello blanco }\end{array}$ & $\begin{array}{l}\text { Semicualificadas } \\
\text { de cuello azul }\end{array}$ & $\begin{array}{l}\text { Ocupaciones } \\
\text { elementales }\end{array}$ & \\
\hline \multirow{4}{*}{$\begin{array}{l}\text { Primarios } \\
0 \text { inferiores }\end{array}$} & Primarios o inferiores & 243,4 & 232,5 & 235,0 & 225,0 & 232,8 \\
\hline & Secundarios & 277,6 & 255,7 & 253,1 & 228,6 & 255,7 \\
\hline & Superiores & 291,5 & 242,2 & 229,9 & 238,8 & 249,1 \\
\hline & Total & 247,8 & 234,8 & 236,0 & 225,5 & 234,5 \\
\hline \multirow[t]{4}{*}{ Secundarios } & Primarios o inferiores & 259,2 & 257,4 & 255,1 & 248,9 & 256,6 \\
\hline & Secundarios & 273,8 & 261,6 & 271,2 & 253,1 & 265,3 \\
\hline & Superiores & 287,6 & 281,5 & 253,1 & 266,1 & 278,1 \\
\hline & Total & 264,1 & 260,1 & 256,8 & 251,4 & 259,7 \\
\hline \multirow[t]{4}{*}{ FPGS/FP \| } & Primarios o inferiores & 274,5 & 262,3 & 264,9 & 246,4 & 265,9 \\
\hline & Secundarios & 270,1 & 267,6 & 281,9 & 281,1 & 271,3 \\
\hline & Superiores & 271,0 & 279,8 & 269,2 & 287,0 & 274,9 \\
\hline & Total & 273,8 & 264,3 & 266,6 & 250,3 & 267,1 \\
\hline \multirow[t]{4}{*}{ Universitarios } & Primarios o inferiores & 290,5 & 284,2 & 254,1 & 261,4 & 287,5 \\
\hline & Secundarios & 292,6 & 278,5 & 277,1 & 255,9 & 288,6 \\
\hline & Superiores & 300,8 & 297,6 & 299,4 & 202,8 & 300,0 \\
\hline & Primarios o inferiores & 293,9 & 285,1 & 274,7 & 258,0 & 290,9 \\
\hline \multirow[t]{4}{*}{ Total } & Primarios o inferiores & 275,1 & 252,1 & 243,1 & 232,3 & 254,3 \\
\hline & Secundarios & 287,5 & 265,9 & 264,7 & 248,2 & 275,2 \\
\hline & Superiores & 298,8 & 284,8 & 268,6 & 253,8 & 291,8 \\
\hline & Total & 282,1 & 256,5 & 245,6 & 234,4 & 261,2 \\
\hline
\end{tabular}

Fuente: población ocupada entre 25 y 65 años en los microdatos del PIAAC (OCDE, 2012).

solo en el análisis de los universitarios: apreciamos que, a mayor nivel educativo del progenitor, mayor es el rendimiento en lectura, por lo que el mismo nivel educativo puede estar asociado a un grado de competencias diferente según el origen social.

De la tabla anterior, debe destacarse el nivel de competencias de las personas en CINE 1 y en CINE 2, 3 o 4, pues en ellos las diferencias por origen social son mayores. Por tanto, a menor nivel educativo, más relevante es el origen social en las competencias alcanzadas en la vida adulta, una prueba clara de la importancia de la familia en la formación de dichas competencias. El proceso selectivo en educación hace que, en cada nivel educativo adicional, la población que entra sea más homogénea en capacidades, igualándose así las competencias en mayor medida por "arriba» (las diferencias en los universitarios entre origen social alto y bajo son de 12,5 puntos), que por «abajo» (las diferencias son de 21,5 puntos en el nivel educativo más bajo). Por ello, son cada vez más los autores que insisten en los beneficios de la escolarización temprana en la mejora tanto de la igualdad de oportunidades como del nivel medio de competencias de toda la población (Heckman, 2006). 


\section{Discusión}

El análisis de los datos presentados lleva a la conclusión de que es difícil encontrar un patrón consistente entre los indicadores de sobrecualificación y las diferentes características individuales, pues solo en una de ellas la relación es congruente en todos los tipos de sobrecualificación: los años de experiencia en el puesto de trabajo, en consonancia con la teoría del ajuste. Esta falta de consistencia puede ser debida a cuestiones metodológicas o a cuestiones más sustantivas. Desde el punto de vista metodológico, la muestra se reduce considerablemente cuando nos centramos en la subpoblación según nivel de estudios y origen social. Por otro lado, hay dificultades para operacionalizar las variaciones de los títulos educativos, pues son muy heterogéneos. Además, las distintas mediciones de la sobrecualificación pueden estar sujetas a errores de medida, como, por ejemplo, la agrupación de la ocupación a dos dígitos (por limitaciones muestrales), que podría llevar a que, bajo una rúbrica, se agruparan puestos de trabajo con cargas cognitivas muy diferentes.

Desde un punto de vista más sustantivo, los diversos estudios arrojan resultados diferentes para cada tipo de indicador de sobrecualificación, lo que podría indicar que cada tipo de medición recoge dimensiones independientes del problema y, por tanto, es necesaria una aproximación plural. Las limitaciones y posibilidades de cada tipo de medición se detallaron en la introducción.

Desde el punto de vista de la movilidad social, sí detectamos que, a mayor nivel de estudios del padre, menor es la probabilidad de estar sobrecualificado en los indicadores objetivo (al igual que Marqués et al., 2015) y subjetivo, pero no se aprecia relación en la sobrecualificación estadística por años de escolarización. La sobrecualificación por competencias sí muestra relación con el origen social, pero en sentido contrario a lo pronosticado. El estudio realizado desde la aproximación de la movilidad social muestra que esto podría deberse a una mayor infracualificación por competencias entre las personas de alto origen social. Al no tener el título educativo adecuado, las mayores competencias asociadas con su origen familiar no son reconocidas por el mercado de trabajo. Esta sería una prueba a favor de las teorías credencialistas y de la señalización.

Desde la aproximación de la movilidad social, se observa una fuerte relación entre origen social y logro educativo, como es habitual en este tipo de estudios. Pero, una vez que se ha alcanzado el título universitario, el origen social no produce grandes efectos sobre la probabilidad de alcanzar ocupaciones de baja cualificación. Este resultado es congruente con la teoría de Boudon (1983), desarrollada más recientemente por Goldthorpe (2010). Según esta teoría, podemos distinguir dos tipos de mecanismos para explicar el logro educativo. Por un lado, los «efectos primarios", que serían todos aquellos factores que contribuyen a determinar las capacidades individuales asociadas al éxito escolar. Entre los efectos primarios, hay tantos factores individuales (salud, capacidades cognitivas y no cognitivas innatas, etc.) como factores sociales (nivel socioeconómico y cultural de la familia). Estos efectos se hacen sentir en las etapas más tempranas del sistema pedagógico, en las cuales unos niños se diferencian 
Tabla 15. Tipo de ocupación alcanzada por el entrevistado (en \%), según nivel educativo del padre o tutor

\begin{tabular}{|c|c|c|c|c|c|c|c|}
\hline \multirow[b]{2}{*}{$\begin{array}{l}\text { Nivel de estudios } \\
\text { del entrevistado }\end{array}$} & \multirow[b]{2}{*}{$\begin{array}{l}\text { Nivel de estudios } \\
\text { del padre o tutor }\end{array}$} & \multicolumn{6}{|c|}{ Tipo de ocupación del entrevistado } \\
\hline & & Cualificadas & $\begin{array}{l}\text { Semicualificadas } \\
\text { de cuello blanco }\end{array}$ & $\begin{array}{l}\text { Semicualificadas } \\
\text { de cuello azul }\end{array}$ & $\begin{array}{l}\text { Ocupaciones } \\
\text { elmentales }\end{array}$ & $\begin{array}{c}\text { Total } \\
(\%)\end{array}$ & $\begin{array}{l}\text { Total } \\
\text { (N) }\end{array}$ \\
\hline \multirow{4}{*}{$\begin{array}{l}\text { Primarios } \\
0 \text { inferiores }\end{array}$} & Primarios 0 inferiores & $10.5 \%$ & $31.3 \%$ & $34.7 \%$ & $24 \%$ & $100 \%$ & 930 \\
\hline & Secundarios & $14.6 \%$ & $46.0 \%$ & $30.8 \%$ & $9 \%$ & $100 \%$ & 65 \\
\hline & Superiores & $19.9 \%$ & $32.0 \%$ & $13.6 \%$ & $35 \%$ & $100 \%$ & 17 \\
\hline & Total & $10.9 \%$ & $32.3 \%$ & $34.1 \%$ & $22.7 \%$ & $100 \%$ & 1013 \\
\hline \multirow[t]{4}{*}{ Secundarios } & Primarios o inferiores & $26.1 \%$ & $44.1 \%$ & $19.4 \%$ & $10 \%$ & $100 \%$ & 516 \\
\hline & Secundarios & $29.8 \%$ & $44.9 \%$ & $12.5 \%$ & $13 \%$ & $100 \%$ & 102 \\
\hline & Superiores & $29.1 \%$ & $46.4 \%$ & $10.3 \%$ & $14 \%$ & $100 \%$ & 55 \\
\hline & Total & $26.9 \%$ & $44.4 \%$ & $17.6 \%$ & $11.1 \%$ & $100 \%$ & 673 \\
\hline \multirow[t]{4}{*}{ FPGS/FP \| } & Primarios o inferiores & $32.1 \%$ & $36.8 \%$ & $24.9 \%$ & $6 \%$ & $100 \%$ & 246 \\
\hline & Secundarios & $33.4 \%$ & $46.7 \%$ & $18.2 \%$ & $2 \%$ & $100 \%$ & 33 \\
\hline & Superiores & $30.6 \%$ & $39.5 \%$ & $24.5 \%$ & $5 \%$ & $100 \%$ & 21 \\
\hline & Total & $32.1 \%$ & $38.0 \%$ & $24.2 \%$ & $5.6 \%$ & $100 \%$ & 300 \\
\hline \multirow[t]{4}{*}{ Universitarios } & Primarios o inferiores & $71.7 \%$ & $23.3 \%$ & $1.5 \%$ & $4 \%$ & $100 \%$ & 480 \\
\hline & Secundarios & $74.9 \%$ & $20.9 \%$ & $2.1 \%$ & $2 \%$ & $100 \%$ & 179 \\
\hline & Superiores & $84.6 \%$ & $12.5 \%$ & $2.5 \%$ & $0 \%$ & $100 \%$ & 225 \\
\hline & Total & $75.6 \%$ & $20.1 \%$ & $1.9 \%$ & $2.4 \%$ & $100 \%$ & 885 \\
\hline \multirow[t]{4}{*}{ Total } & Primarios o inferiores & $30.2 \%$ & $33.2 \%$ & $22.6 \%$ & $14 \%$ & $100 \%$ & 2173 \\
\hline & Secundarios & $48.9 \%$ & $33.9 \%$ & $11.2 \%$ & $6 \%$ & $100 \%$ & 379 \\
\hline & Superiores & $68.0 \%$ & $21.2 \%$ & $5.9 \%$ & $5 \%$ & $100 \%$ & 318 \\
\hline & Total & $36.8 \%$ & $32.0 \%$ & $19.3 \%$ & $12.0 \%$ & $100 \%$ & 2870 \\
\hline
\end{tabular}

Fuente: población ocupada entre 25 y 65 años en los microdatos del PIAAC (OCDE, 2012).

de otros por su facilidad para mostrar un buen rendimiento académico. Pero, debido a la selección producida al final de cada etapa educativa, cuanto mayor sea esta, menor será el peso de los efectos primarios, pues el alumnado se va igualando por rendimiento. Por ello, las diferencias en competencias por origen social son pequeñas a igualdad de nivel educativo (tabla 14), aunque son considerables en cuanto a la probabilidad de alcanzar cierto nivel formativo (tabla 15).

Los datos apuntan, por tanto, a que el mayor peso en la explicación de la desigualdad social está en la relación entre familia y logro académico, y en mucha menor medida, en la relación entre sistema educativo y mercado de trabajo. Los datos, por tanto, no apoyan a las tesis de Bourdieu (1991) o a las más recientes de Goldthorpe (en el trabajo citado con Bukodi), según las cuales los factores no cognitivos desempeñan un papel importante en la movilidad social. Ambos autores se refieren especialmente a un contexto en el que se haya expandido considerablemente la educación superior. Para controlar este hecho, se procedió a realizar estimaciones solo para las personas entre 30 y 45 años, protagonistas del «boom de la universidad» en España, sin que las conclusiones 
sean diferentes. Incluso podría suceder al contrario de lo que proponen ambos autores, pues las personas que provienen de un origen social bajo con titulación universitaria manifiestan desempeñar ocupaciones con más carga de factores no cognitivos. Una hipótesis a explorar es que, a diferencia de otros países, la expansión universitaria ha sido más tardía y rápida en España, por lo que el contexto histórico no es comparable al de Francia o del Reino Unido.

\section{Conclusiones}

El objeto de este análisis ha sido establecer una relación entre sobrecualificación y movilidad social, con el objeto de contrastar si el origen social puede estar relacionado con la sobrecualificación y en qué medida su relación puede ser mayor o menor que la de otros factores asociados a la sobrecualificación, tales como el sexo, la edad, la nacionalidad o la experiencia laboral. Para ello, se han adaptado a los datos del PIAAC tres indicadores habituales en la literatura sobre esta cuestión, a los que se han añadido dos nuevos, gracias a la riqueza de información de este estudio. La investigación se ha centrado especialmente en el caso de la sobrecualificación de los titulados universitarios.

La relación entre los distintos indicadores de sobrecualificación y las características estudiadas de los ocupados no es consistente, es decir, una misma característica puede estar asociada a efectos de signo contrario en distintos indicadores. Esto podría deberse tanto a problemas metodológicos como a cuestiones más sustantivas, en el sentido de que cada indicador refleja una dimensión distinta del problema estudiado.

Se ha probado una aproximación diferente a la sobrecualificación, a partir de la agrupación en cuatro de las ocupaciones (cualificadas, semicualificadas de cuello blanco, de cuello azul y elementales), lo cual metodológicamente nos aparta de los análisis estándar de la sobrecualificación y nos acerca más a la tradición de investigación sobre movilidad social. Tras comprobar la intensa relación entre origen social (medido por los estudios del padre) y nivel educativo de los entrevistados, se ha detectado otra relación más débil: entre quienes tienen título universitario, los de origen social alto es más probable que desempeñen ocupaciones cualificadas. También sucede que el nivel de competencias en lectura de las personas de origen social alto es un poco más elevado, lo cual podría explicar el mejor ajuste de los universitarios, debido en parte a que entre ellos abundan más los licenciados que los diplomados, y que su nivel de competencias en lectura es 10 puntos superior al resto.

Estos resultados sugieren que el mayor peso de la desigualdad de oportunidades está en la relación entre origen social y rendimiento académico, y no tanto en la relación entre origen social y mercado de trabajo. La mejora en la igualdad de oportunidades, por tanto, debería descansar más sobre políticas educativas que laborales. 


\section{Anexo}

Gráfico 1. Gráfico en espacio rotado de los factores extraídos por componentes principales

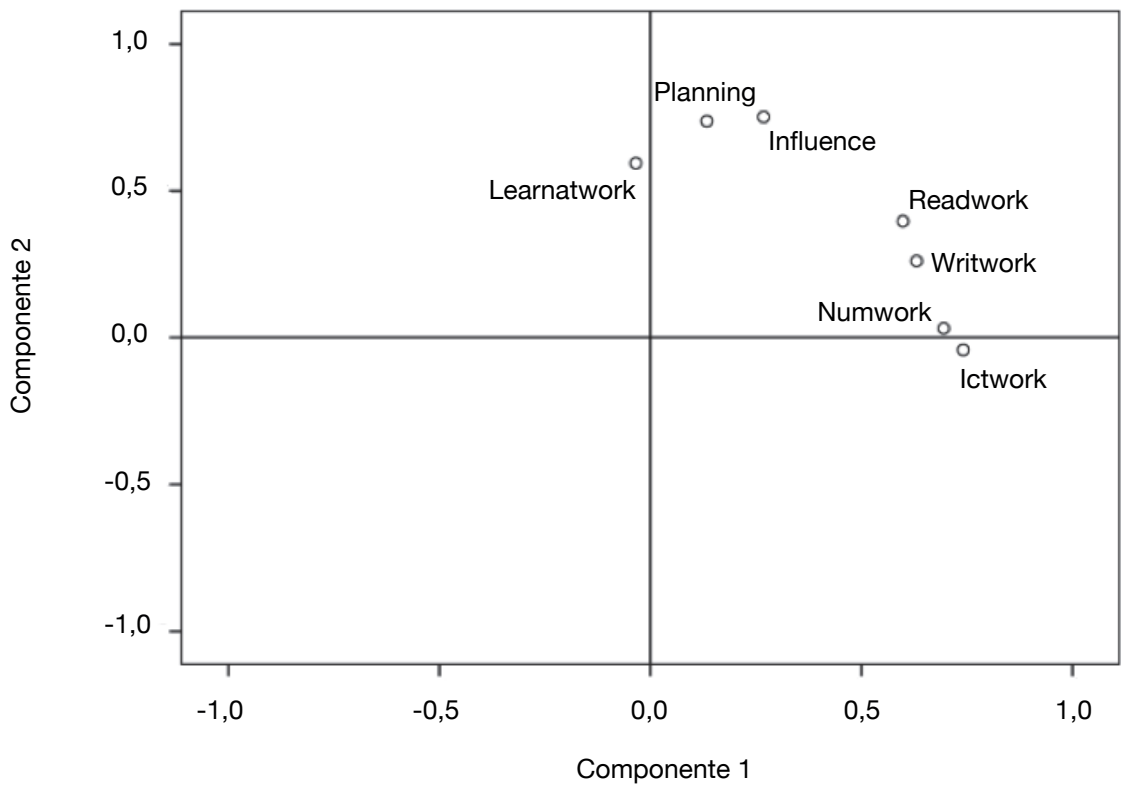

Calculado solo para quienes trabajaron con remuneración la semana anterior a la encuesta. Los casos perdidos han sido sustituidos por valores medios.

\section{Referencias bibliográficas}

Barone, Carlo y Ortiz, Luis (2011). «Overeducation among European University Graduates: A comparative analysis of its incidence and the importance of higher education differentiation». Higher Education [en línea], 61, 325-337. <http://dx.doi.org/10.1007/s10734-010-9380-0>.

Becker, Gary S. (1964). Human capital: A theoretical and empirical analysis, with special reference to education. Nueva York: Columbia University Press.

Boudon, Raymond (1983). La desigualdad de oportunidades. Barcelona: Laia.

Bourdieu, Pierre (1991). La distinción. Madrid: Taurus.

Bukodi, Erzsebet y Goldthorpe, John (2011). «Social class returns to higher education: Chances of access to the professional and managerial salariat for men in three British birth cohorts». Longitudinal and Life Course Studies [en línea], 2. <http://dx.doi.org/10.14301/llcs.v2i2.122>.

Cabrales, Antonio; Dolado, Juan José y Mora, R. (2013). "Dualidad laboral y déficit de formación ocupacional: Evidencia sobre España con datos de PIAAC». En: Informe nacional del Programa Internacional de Evaluación de Competencias de la Población Adulta, tomo II. Madrid: Ministerio de Educación y Cultura. Instituto Nacional de Evaluación Educativa, 9-38. 
Capsada-Munsech, Queralt (2015). "The role of social origin and field of study on graduates' overeducation: The case of Italy». Higher Education [en línea], 69, $779-807$. <http://dx.doi.org/10.1007/s10734-014-9805-2>.

DesJARDINS, Richard y WARNKE, Jonas (2011). «Ageing and Skills: A review and analysis of skill gain and skill loss over the lifespan and over time». OECD Working Paper.

Dolado, Juan J.; Felgueroso, Florentino y Jimeno, Juan F. (2000). «Youth labour markets in Spain: Education, training, and crowding-out». European Economic Review [en línea], 44, 943-956.

<http://dx.doi.org/10.1016/s0014-2921(99)00050-1>.

Fernández Mellizo-Soto, María (2014). «Desigualdad de oportunidades educativas en España». Reis, 147, 107-20. <http://dx.doi.org/10.5477/cis/reis.147.107>.

Flisi, Sara; Goglio, Valentina; Meroni, Elena Claudia; Rodrigues, Margarida y Vera-Toscano, Esperanza (2016). «Measuring Occupational Mismatch: Overeducation and Overskill in Europe-Evidence from PIAAC». Social Indicators Research [en línea], 1-39. <http://dx.doi.org/10.1007/s11205-016-1292-7>.

Freeman, R.B. (1976). The overeducated american. Nueva York: Academic Press.

García Montalvo, José y Peiró, José M. a (2009). Análisis de la sobrecualificación y la flexibilidad laboral. Valencia: IVIE.

Glebbeek, A. C. (1993). Perspectieven op Loopbanen. Assen: Van Gocum.

GoldThorpe, John H. (2010). Sobre la sociología. Madrid: CIS.

Heckman, James J. (2006). «Skill Formation and the Economics of Investing in Disadvantaged Children». Science [en línea], 312, 1900-1903. <http://dx.doi.org/10.1126/science.1128898>.

KucEL, Aleksander (2010). «The sociology of educational mismatch». DemoSoc Working Papers. Barcelona: Universidad Pompeu Fabra.

- (2011). "Literature Survey of the Incidence of Over-education: A Sociological Approach». Revista Española de Investigaciones Sociológicas, 134, 125-142.

Leuven, Edwin y Oosterbeek, Hessel (2011). "Overeducation and mismatch in the labor market». IZA Discussion Paper.

McGuinness, Seamus y Wooden, Mark (2007). "Overskilling, job insecurity and career mobility». IZA Discussion Paper 2938.

Marqués, Ildefonso y Gil, Carlos (2015). «Origen social y sobreeducación en los universitarios españoles». REIS, 150, 89-112. $<$ http://dx.doi.org/10.5477/cis/reis.150.89>.

Martín Moreno, J. y Miguel, A. de (1979). Universidad, fábrica de parados. Barcelona: Vicens Vives.

Martínez García, José Saturnino (2011). «Género y origen social: Diferencias grandes en fracaso escolar y bajas en rendimiento educativo". Revista de la Asociación de Sociología de la Educación, 4, 270-285.

Mincer, Jacob (1974). Schooling, experience and earnings. Nueva York: Columbia University Press.

OCDE (2012). Microdatos del Proyecto Internacional de Competencias de la Población Adulta (PIAAC). Consulta 10/11/16 de URL: <http://www.oecd.org/skills/piaac/publicdataandanalysis>.

OCDE (2013). OECD skills outlook 2013: First results from the survey of adult skills. París: OCDE. 
Pissarides, Christopher (2000). Equilibrium Unemployment Theory. Cambridge (MA): MIT Press.

Quintini, Glenda (2011). Over-Qualified or Under-Skilled: A Review of Existing Literature. París: OECD.

SALA, Guillermo (2011). "Approaches to Skills Mismatch in the Labour Market: A literature review». Papers, 96 (4), 1025-1045. < http://dx.doi.org/10.5565/rev/papers/v96n4.171>.

Sattinger, Michael (1993). «Assignment Models of the Distribution of Earnings». Journal of Economic Literature, 31, 851-880.

Sloane, Peter J. (2003). «Much Ado About Nothing?: What Does the Over-education Literature Really Tell us?». En: Buchel, F.; DeGrip, A. y Mertens, A. (ed.). Overeducation in Europe: Current Issues in Theory and Practice. Northampton: Edward Elgar.

Spence, Michael (1973). "Job market signaling». The Quarterly Journal of Economics [en línea], 87, 355-374. <http://dx.doi.org/10.2307/1882010>.

Thurow, Lester C. (1975). Generating inequality: Mechanisms of distribution in the US economy. New York: Basic Books.

Torche, Florencia (2011). «Is a College Degree Still the Great Equalizer?: Intergenerational Mobility across Levels of Schooling in the United States». American Journal of Sociology [en línea], 117, 763-807. <http://dx.doi.org/10.1086/661904>.

Treiman, Donald J. (1970). «Industrialization and Social Stratification». En: LauNMANn, E.O. (ed.). Social Stratification Research and Theory for the 1970s. Indianapolis: Bobbs-Merill. 